\title{
Research on 3D Self-Stable Pan-Tilt Structural Control Methods
}

\author{
Pengpeng Yang ${ }^{1}$, Xingpan $\mathrm{Cui}^{1}$ \\ ${ }^{1}$ Tianjin University of Technology and Education, Tianjin University of Technology and Education No.1310,Dagu South Road, Hexi District, \\ Tianjin 300222, China
}

\begin{abstract}
With the development of society, flexible cable-driven camera robot system has found wide applications. Self-stable pan-tilt is an indispensable part of the end effector. To obtain quality and authentic images, the end effector should stably move at a constant speed and the optical axis of the camera of the effector should scan the ground evenly. However, a series of factors, such as changing load of the effector, influence of the wind resisting moment and the sensor noise, might distort images obtained, thus influencing the photographing quality. In order to achieve tracking shooting of the target under stable conditions of the camera, it is necessary to optimize the system hardware on the one hand; improve the system control accuracy through the modern control algorithm on the other hand.
\end{abstract}

Keywords: Self-stable pan-tilt, Mathematical model, PID, Fuzzy PID, Simulation

\section{Introduction}

Self-stable pan-tilt is a carrier of cameras, which is used to insulate disturbance, such as changing postures of the end effector, system vibration and wind resistance, so as to ensure stability of the optical axis of the end effector [1, 2]. With the self-stable pan-tilt, the efficiency of the aerial survey and the quality of remote sensing images can be increased, the working intensity of workers can be alleviated and the influence of weather and other environmental factors on shooting can be weakened so as to improve the shooting effect and the post-processing quality.

\section{Mathematical Model}

Multi-platform The 3D self-stable pan-tilt structure is mainly made up of three mutually vertical frames, namely the external frame, the middle frame and the internal frame[3]. The external frame is used to mainly control up and down focusing; the middle frame is mainly responsible for controlling direction and position of the camera; and the internal frame is in charge of controlling the rotation of the camera. During the shooting process, when some interfering factors impede stable shooting, the position sensor above the end effector can measure the positional deviation of the camera and send the deviation through the telecommunication cable to the control center. The control center will generate corresponding control signals through calculation, and send these signals to the servo motor to make the electromotor twist, which can counteract the positional deviation of the camera. In this way, the camera can stably come up with clear images. Below is the structural chart of the 3D head support:

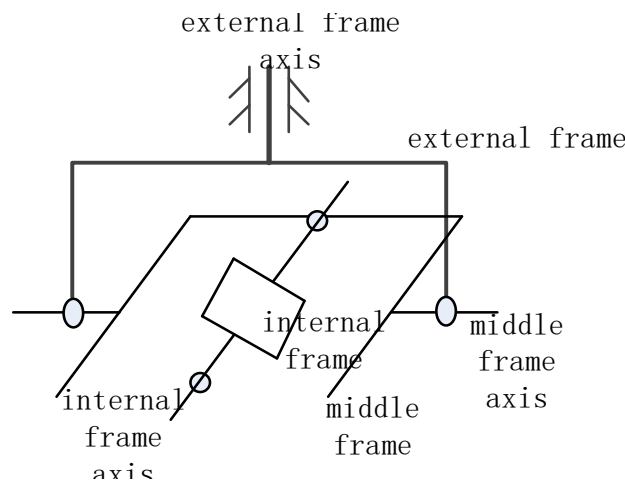

Figure1: Structural chart of the 3D self-stable pan-tilt

The kinematic equation of every channel of the 3D head support can be obtained based on parameters in literatures: Pitching channel:

$$
G(S)=\frac{16470}{S^{2}+1667 S+3393}
$$

Rolling channel:

$$
G(S)=\frac{14570}{S^{2}+2000 S+11070}
$$

Positional channel:

$$
G(S)=\frac{13060}{S^{2}+1667 S+3590}
$$

\section{PID Theory}

The PID controller is made up of P (the proportion element), I (the integral element) and D (the derivative element) [4]. The functional block diagram of the continuous-time PID control within the time domain is shown below: 


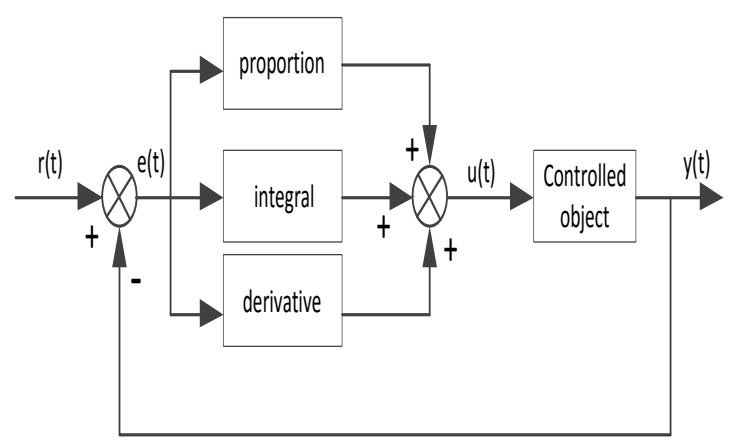

Figure2: PID control system principle diagram

Functions of three parameters, proportion, integral and derivative are shown below:

\subsection{Functions of the proportion}

The transfer function of the proportion control rule is shown below:

$$
u(t)=k_{p} e(t)
$$

To enlarge signals is the core function of the proportion adjuster. To enlarge the deviation signal, e $(t)$, is the major function of the proportional controller. When there is deviation, the controller can quickly control it to reduce the deviation to the minimum. When $\mathrm{kp}$ is too large, it will result in instability of the loop system since the relative stability of the system is significantly reduced even if the system's steady state error is reduced to the minimum. The smaller $\mathrm{kp}$ is, the higher the steady state error is. However, this is favorable for stability maintenance, which can avoid emergence of dramatic turbulences.

\subsection{Functions of the integral}

The transfer function of the proportion control rule is shown below:

$$
u(t)=\frac{1}{T_{i}} \int e(t) d t
$$

The major function of the integral control is to eliminate the steady state error. As long as there is deviation between the target value and the feedback value of the system, the effector will keep on moving. The stronger the deviation control is, the faster the movement speed, $\mathrm{u}(\mathrm{t})$, of the effector output is. The weaker the deviation control is, the smaller the movement speed, u (t), of the output of the effector is. When the deviation is zero, the effector cannot output anything and the control function ends. At the moment the control system can reach a balance.

\subsection{Functions of the derivative}

The transfer function of the proportion control rule is shown below:

$$
u(t)=T_{d} \frac{d e(t)}{d t}
$$

Major characteristics of the derivative control: The derivative control is related to $\mathrm{u}(\mathrm{t})$ and the derivation, e (t). It has nothing to do with derivation. The changing rend of the derivative signals is decided by the derivate control. The derivation signal value can be controlled in advance.

\subsection{PID Simulation Analysis}

In this paper, the author adopts the kinematic equation of the external frame as an example for simulation:

$$
G(S)=\frac{16470}{S^{2}+1667 S+3393}
$$

As to three parameters $\mathrm{kp}=40, \mathrm{ki}=30, \mathrm{kd}=0.011$, used during the simulation process, the Ziegler-Nichols rectification method can be used to confirm them. The input signals are the unit step signals. Below are simulation results:

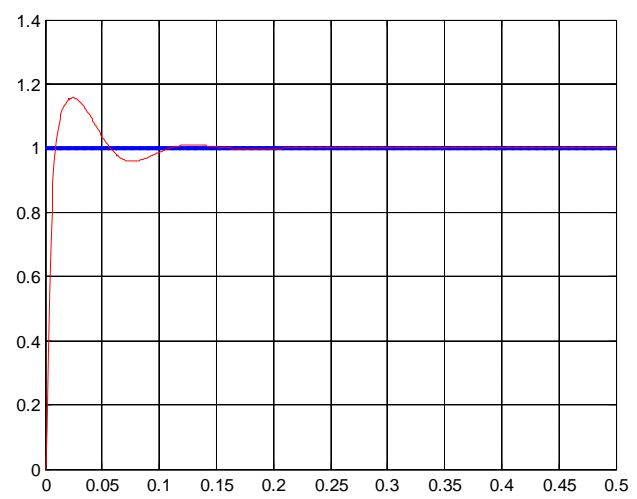

Figure 3: PID control simulation curve

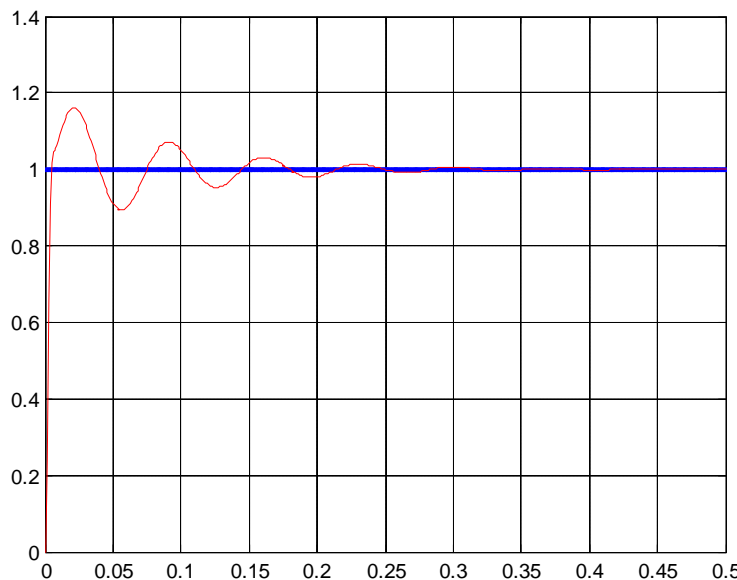

Figure 4: PID control simulation curve under interference

As is shown about figure 3 , the system adjustment takes about 0.15 s under the condition of no interfering factors. There is almost no steady state error and the overshooting, which can basically meet the control requirements. 


\section{International Journal of Science and Research (IJSR) \\ ISSN (Online): 2319-7064 \\ Index Copernicus Value (2013): 6.14 | Impact Factor (2014): 5.611}

As is shown about figure 4, when the noise interference is introduced to the system, the system will undergo dramatic vibration. Thus, it can be seen that the traditional PID control cannot well control the inhibition effect.

Based on analysis of the simulation results, it can be seen that: when there is no noise interference, the adjustment speed of the traditional PID control is fast and the steady state error is small, which can generally meet the system control requirements. However, affected by the noise interference, the traditional PID control effect is insignificant. In order to help system to achieve a favorable effect under the situation of noise interference, it is imperative to improve the traditional PID control.

\section{Fuzzy Controller}

Generally speaking, the fuzzy controller is made up of four parts, namely the fuzzy interface, the fuzzy rules, the fuzzy logic computer and the fuzziness-solving interface $[5,6]$. The basic structure of the fuzzy controller is shown below:

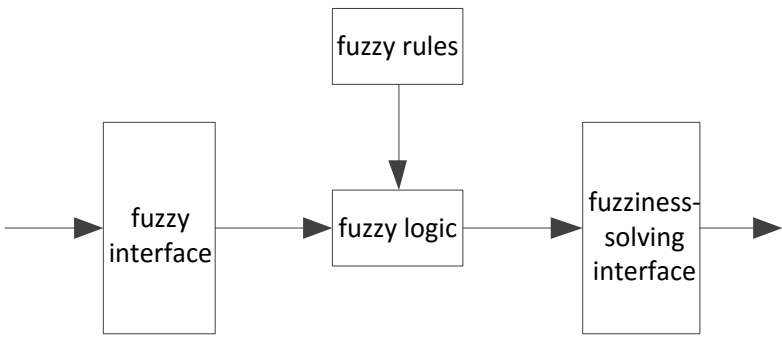

Figure 5: Basic structural chart of the fuzzy controller

The role of the fuzzy interface is to transfer an accurate digital quantity into a fuzzy linguistic value applicable to the fuzzy algorithm. The design of the fuzzy interface mainly consists of four steps, namely confirmation of language variables, design of language variable domain of discourse, definition of the linguistic value of various language variables and definition of the membership function of different linguistic value.

Extraction of fuzzy rules is the most important step during the design process of the fuzzy controller. The extraction quality directly decides whether control effects of the fuzzy controller can meet requirements.

Fuzzy reasoning is to solve a series of fuzzy relation equations and obtain the fuzzy value output by the fuzzy controller according to the fuzzy quantity output by the system and to the fuzzy rules obtained.

The fuzziness-solving interface is to transfer the fuzzy control quantity into the accurate digital quantity for system control. There are mainly two fuzziness solutions, namely the maximum membership degree and the weighted average method.

\subsection{Design of the fuzzy PID controller}

The fuzzy PID control is to apply the fuzzy control algorithm to the typical PID controller to obtain an improved technique. The principle of the process is to first transfer control conditions and rules into utilizable fuzzy language in the fuzzy algorithm, and then store these fuzzy rules into the computer knowledge bank. The computer will choose suitable control rules from the knowledge bank and automatically adjust parameters according to the system response.

The fuzzy PID control is improved based on the traditional PID control. It significantly makes up for defects of the traditional PID. First, it can obviously improve the system's adjustment speed and increase the robustness of the control system. Second, the system's control results can be more accurate, and the system's control performance can be greatly enhanced. The structural chart of the fuzzy PID controller is shown below:

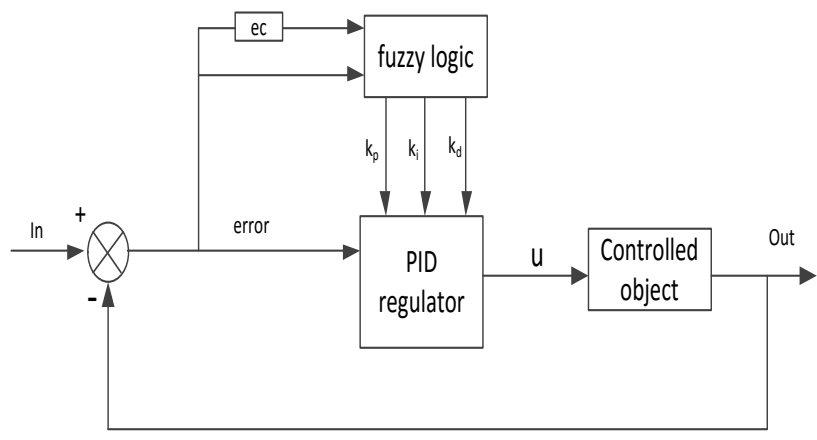

Figure 6: Structural chart of the fuzzy PID controller

From the structural chart, it can be seen that the parameter setting of the fuzzy PID control is to seek the fuzzy relationship among parameters ( $\mathrm{kp}$, ki and kd), the error, e, and the error change rate ec. During system operation, e and ec will be constantly tested. According to the agreed relationship, the fuzzy reasoning can be adopted to automatically realize the optimal adjustment of parameters of the PID controller.

\subsection{Fuzzy PID simulation analysis}

Similarly, the kinematic equation of the external frame is adopted for simulation. The PID parameter value setting is the same to the above. The unit step signals are input. The sampling time is $1 \mathrm{~ms}$. The simulation results are shown below:

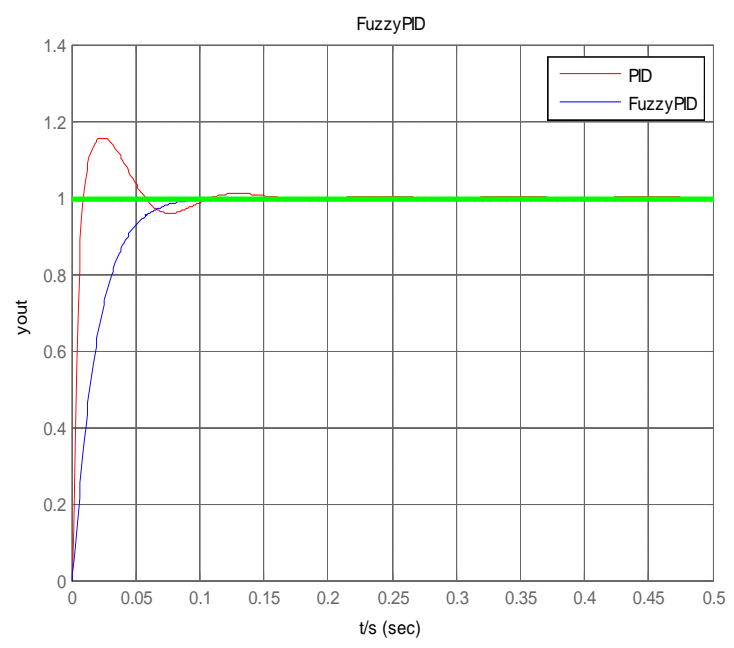

Figure 7: The fuzzy PID control simulation curve 
As is shown above, the adjustment time of the fuzzy PID control is obviously shorter than the traditional PID control. The former takes only $0.07 \mathrm{~s}$, and the control process is relatively stable almost without any overshooting. Thus, it can be seen that the fuzzy PID control performance is significantly superior to the traditional PID control, which can quickly stabilize the system, but might cause huge steady state error. Therefore, further optimization is required to increase the system accuracy.

\section{References}

[1] Manfred Hiller, Shiqing Fang, Sonja Mielczarek, Richard Verhoeven, Daniel Franitza. Design, analysis and realization of tendon-based parallel manipulators [J]. Mechanism and Machine Theory, 2005, 40(4):42- 45.

[2] TAGHIRAD H D, NAHON M A. Forward kinematics of a macro-micro parallel manipulator. IEEE/ASME International Conference on Advanced Intelligent Mechatronics, AIM, Piscataway: IEEE Inc.2007.

[3] Slobodan N, Vukosavic. Suppression of Torsional Oscillations in A High-Performance Speed Servo Drive [J]. IEEE Trans. on Industry Electronics. 1998, 45(1): 108-117.

[4] KON, WU J. APSO-tuning method for design of fuzzy PID controllers [J]. Journal of Vibration and Control, 2007, 12: 1-18.

[5] Bo Li, D Hullender, M De Renzo. Nonlinear Induced Disturbance Rejection in Inertial Stabilization Systems [J].IEEE Trans. on Control System Technology. 1998, 6(3): 421-427.

[6] Marcelo C A, Douglas E E. Novel Kalman Filtering Method for the Suppression of Gyroscope Noise Effects in Pointing and Tracking Systems[J]. Optical engineering. 1995, 34(10): 3016-3030.

\section{Author Profile}

Pengpeng Yang is reading the M.S. degrees in Mechanical Engineering from Tianjin University of Technology and Education.

Xingpan Cui is reading the M.S. degrees in Mechanical Engineering from Tianjin University of Technology and Education. 